Article

\title{
Posterior Wnts Have Distinct Roles in Specification and Patterning of the Planarian Posterior Region
}

\author{
Miquel Sureda-Gómez, Eudald Pascual-Carreras and Teresa Adell * \\ Received: 20 September 2015 ; Accepted: 28 October 2015 ; Published: 5 November 2015 \\ Academic Editor: Francesc Cebrià \\ Department of Genetics and Institute of Biomedicine, University of Barcelona, Barcelona E-08028, Catalonia, \\ Spain; msureda6@gmail.com (M.S.-G.); eudald.pascual@gmail.com (E.P.-C.) \\ * Correspondence: tadellc@ub.edu; Tel.: +34-93-402-15-00
}

\begin{abstract}
The wnt signaling pathway is an intercellular communication mechanism essential in cell-fate specification, tissue patterning and regional-identity specification. A $\beta$ catenin-dependent signal specifies the AP (Anteroposterior) axis of planarians, both during regeneration of new tissues and during normal homeostasis. Accordingly, four wnts (posterior wnts) are expressed in a nested manner in central and posterior regions of planarians. We have analyzed the specific role of each posterior wnt and the possible cooperation between them in specifying and patterning planarian central and posterior regions. We show that each posterior wnt exerts a distinct role during re-specification and maintenance of the central and posterior planarian regions, and that the integration of the different wnt signals ( $\beta$ catenin dependent and independent) underlies the patterning of the AP axis from the central region to the tip of the tail. Based on these findings and data from the literature, we propose a model for patterning the planarian AP axis.
\end{abstract}

Keywords: patterning; identity specification; wnt signaling; planarians

\section{Introduction}

The wnt signaling pathway is an intercellular communication mechanism with essential roles in cell-fate specification, tissue patterning and specification of regional identity [1]. Wnts, the secreted elements of the pathway, interact with the membrane receptors Frizzleds and their co-receptors (LRP4/5, Ror, Ryk) to transduce different signals that branch mainly in three pathways: the canonical or $\beta$ catenin-dependent wnt signaling, and two non-canonical or $\beta$ catenin-independent signals, which regulate either JNK (c-Jun N-terminal kinase) or PKC (Protein kinase C) pathways [1-3]. The $\beta$ catenin-dependent pathway exerts its function by regulating the nuclear translocation of $\beta$ catenin, and is mainly involved in cell-fate specification. One of the most conserved roles of ßcatenin-dependent Wnt signaling is the specification of the AP (Anteroposterior) axis, where Bcatenin is required to confer posterior features in most developmental models studied [4]. $\beta$ catenin-independent pathways are mainly involved in the control of cell shape and movements $[5,6]$.

Planarians are an ideal model for the study of cell-fate specification and patterning, since they are extremely plastic. They are bilateral animals with a complex cephalized nervous system and a three-branched gut which converges into a pharynx, which takes in food and expulses debris through a ventral mouth [7]. Planarians can regenerate any amputated part, even the head, in a few days, and they continuously remodel their tissues while they grow and shrink according to food availability and temperature. Those capabilities are due to the presence of a population of totipotent stem cells all around their body, the neoblasts, which are able to differentiate to any cell type [7-9]. Because of its astonishing regenerative abilities, planarians have been established as a unique model to understand stem cell biology and the molecular mechanisms underlying patterning and 
regional identity specification. Specifically, the function of the Wnt signaling pathway has been extensively studied in planarians [10-19]. Due to its plasticity, even in the adult stage, the phenotypes generated when silencing Wnt pathway elements had no precedent in the field of developmental

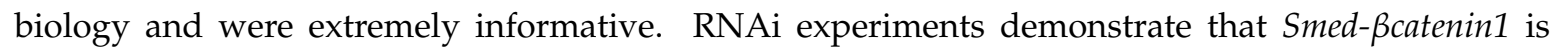
essential to pattern the AP axis in planarians, since its inhibition generates anteriorized phenotypes ranging from "tailless" planarians to "two-headed" planarians and, most strikingly, "radial-like hypercephalized" planarians [12,20]. Moreover, the study of several elements of the pathway confirms this function, since inhibition of $A P C$ and axin, elements of the $\beta$ catenin destruction complex, lead to posteriorization of planarians $[11,18]$. Interestingly, the Smed- $\beta$ catenin1-dependent Wnt signal is required to specify AP identities both during planarian regeneration and during homeostasis $[12,17,18]$.

Consistent with the role of the $\beta$ catenin-dependent Wnt signal in AP axial specification, 4 wnts are expressed in the posterior part of planarians in a nested manner, which we name in this study posterior wnts (Smed-wnt1, Smed-wnt11-1, Smed-wnt11-2 and Smed-wnt11-5) [15,17-19]. Since planarians such as $S$. mediterranea typically measure at least $1-2 \mathrm{~mm}$ in length, the field is too large to be patterned by a single morphogen. It has therefore been proposed that cooperation between posterior wnts could be required to pattern the AP axis [20]. Out of the four posterior wnts, however, only Smed-wnt1 and Smed-wnt11-2 have been studied functionally. During regeneration of the tail, Smed-wnt1 inhibition leads to "tailless" or "two-headed" planarians, and Smed-wnt11-2 inhibition leads to "tailless" planarians $[14,15,19]$. Although those two wnts seem to be regulators of $\beta$ catenin activity, because its silencing produces an anteriorized phenotype, the strong anteriorization of planarians produced after Smed-Bcatenin 1 silencing has never been phenocopied by the inhibition of any wnt. The purpose of the present study is to analyze the specific role of each posterior wnt and the possible cooperation among them both during regeneration and maintenance of the AP axis. Our data demonstrates that each posterior wnt exerts a distinct function during posterior regeneration, and that the inhibition of all of them generates a stronger anteriorization than the inhibition of any of them alone. During homeostasis, simultaneous silencing of the four posterior wnts also generates a stronger phenotype than silencing any wnt alone, although a shift of posterior to anterior identity is never achieved. We conclude that the integration of the different Wnt signals ( $\beta$ catenin dependent and independent) underlies the patterning of the AP axis from the central region to the tip of the tail.

\section{Results}

\subsection{Individual Posterior Wnts Exert Specific Roles during Posterior Regeneration}

To study the role of each posterior wnt during posterior regeneration, we first analyzed their expression pattern by in situ hybridization. In agreement with previous reports, the four posterior wnts are found to be expressed in a graded manner along the AP axis in intact planarians (Figure S1A) [19]. Smed-wnt1 expression is restricted to few cells in the posterior midline; Smed-wnt11-1 and Smed-wnt11-2 are expressed from the mouth to the tip of the tail, and Smed-wnt11-1 also in the mouth itself; and Smed-wnt11-5 is expressed from the pre-pharyngeal region to the tip of the tail. Interestingly, all of them are expressed as a gradient, higher in the most posterior tip. Moreover, posterior whts are also expressed in a temporal manner during posterior regeneration, being Smed-wnt1 the first one, expressed few hours after cutting (Figure S1B) [14,19], followed by Smed-wnt11-1 and Smed-wnt11-2, which are detected 2 days after cutting (Figure S1B) [19]. Smed-wnt11-5 is expressed at all regeneration stages, since its expression is not lost after cutting the tail but just re-patterned (Figure S1B) [19]. Those expression patterns suggest that each posterior wnt could exert a specific role during posterior specification and patterning, and that the cooperation between them could enable a correct and complete posterior pattern.

To test the specific role of each posterior wnt, we analyzed the morphology and pattern of the tail regenerated by planarians in which each posterior wnt alone was silenced. Phenotypes 
were analyzed by morphological observation and by immnohistochemistry with anti-synapsin and anti- $\beta$ catenin 2 antibodies, to visualize the nervous and the digestive system, respectively (Figure 1 and Figure S2). As expected, inhibition of Smed-wnt1 led to "tailless" and "two-headed" planarians (Figure 1A and Figure S1). Immunohistochemical analysis showed that "two-headed" planarians always differentiate a second pharynx in the opposite direction to the original one, according to the new axis generated in the posterior tip (Figure $1 \mathrm{~A}\left(\mathrm{D}^{\prime}\right)$ ). "Tailless" planarians showed a rounded closure of ventral nerve cords (VNCs) and an undefined posterior tip (Figure $1 \mathrm{~A}\left(\mathrm{~B}^{\prime}, \mathrm{C}^{\prime}\right)$ ) [15]. Among "tailless" planarians two different phenotypes could be distinguished: animals in which only one pharynx was observed (sometimes in an opposite orientation) (Figure $1 \mathrm{~A}\left(\mathrm{~B}^{\prime}\right)$ ) and animals in which two pharynges in opposite orientation could be observed (Figure $1 \mathrm{~A}\left(\mathrm{C}^{\prime}\right)$ ). Silencing of Smed-wnt11-1 lead to the regeneration of shorter tails, in which the distance from the pharynx to the posterior tip was clearly shorter (Figure 1 and Figure S2). Immunohistochemical analysis showed that those animals close properly the VNCs in the posterior tip, and no signal of anteriorization can be observed (Figure $1 \mathrm{~A}\left(\mathrm{E}^{\prime}\right)$ ). Again, two different phenotypes could be distinguished when analyzing the central region, since in some animals a second pharynx appeared in parallel and very close to the pre-existing one (Figure 1A(F)). Interestingly, two-pharynged Smed-wnt11-1 RNAi animals never showed two mouths (Figure $1 \mathrm{~A}\left(\mathrm{~F}^{\prime \prime}\right)$ ). Silencing of Smed-wnt11-2 always lead to the regeneration of "tailless" planarians, as had been already reported (Figure 1 and Figure S1) [15,19]. Immunohistochemical analysis demonstrated that although Smed-wnt11-2 RNAi animals only show the normal pre-existing pharynx, a second mouth appears in half of the animals (Figure $1 A\left(G^{\prime}, G^{\prime \prime}\right)$ ). A second pharynx associated to the second mouth has not been observed, although in few cases a pharynx primordium could be guessed (Figure 1A( $\left.\mathrm{H}^{\prime}\right)$ ). Smed-wnt11-5 RNAi animals apparently regenerated a perfect tail (Figure S2). Immunohistochemical analysis corroborates that VNCs close normally in the posterior tip. However, in most of the cases a second pharynx, oriented either in the same or in opposite direction with respect to the original one, can be observed (Figure $1 \mathrm{~A}\left(\mathrm{I}^{\prime}, \mathrm{J}^{\prime}\right)$ ). A second mouth always differentiates associated to the second pharynx (Figure 1A $\left(\mathrm{I}^{\prime \prime}\right)$ ). Thus, in Smed-wnt11-5 RNAi animals, posterior identity appears normal but the central region appears duplicated. The quantification of the different phenotypes observed after silencing each posterior wnt alone allows the visualization of the different degrees of anteriorization generated (Figure 1B).

We then analyzed posterior identity specification of planarians in which posterior wnts were silenced. Posterior wnts and $f z 4$ were used as markers [18]. Results show that after Smed-wnt1 RNAi the rest of posterior wnts and $f z 4$ disappear or decrease significantly, demonstrating the loss of posterior identity in "tailless" and "two-headed" phenotypes (Figure 1C). In contrast, after Smed-wnt11-1 and Smed-wnt11-5 RNAi, all posterior markers were expressed in the same pattern and levels as in controls, in agreement with the normal posterior closure of the VNCs in the posterior tip (Figure 1C). Thus, Smed-wnt11-1 and Smed-wnt11-5 RNAi animals have normal posterior identity. Smed-wnt11-2 RNAi animals displayed a significant decrease in the expression of posterior markers, according to the "tailless" phenotype observed. Expression of Smed-wnt1 appeared not only diminished but totally disorganized (Figure 1C) [19]. Interestingly, expression of Smed-wnt11-1 and Smed-wnt11-2 was found to be dependent on Smed-wnt1, although it remains unclear whether this is a direct regulation or a consequence of the loss of posterior identity.

Taken together, these results suggest that Smed-wnt1 and Smed-wnt11-2 specify posterior identity, although only Smed-wnt1 RNAi animals exhibit a shift in polarity. Moreover, Smed-wnt11-2 exerts a role in patterning or specifying central identity, since its inhibition duplicates the mouth. Smed-wnt11-1 and Smed-wnt11-5 are not required to specify the identity of the posterior tip. However, they have a role in patterning or specifying the central region, since ectopic pharynges differentiate when they are silenced. Smed-wnt11-1 would be also required to properly elongate the tail. 

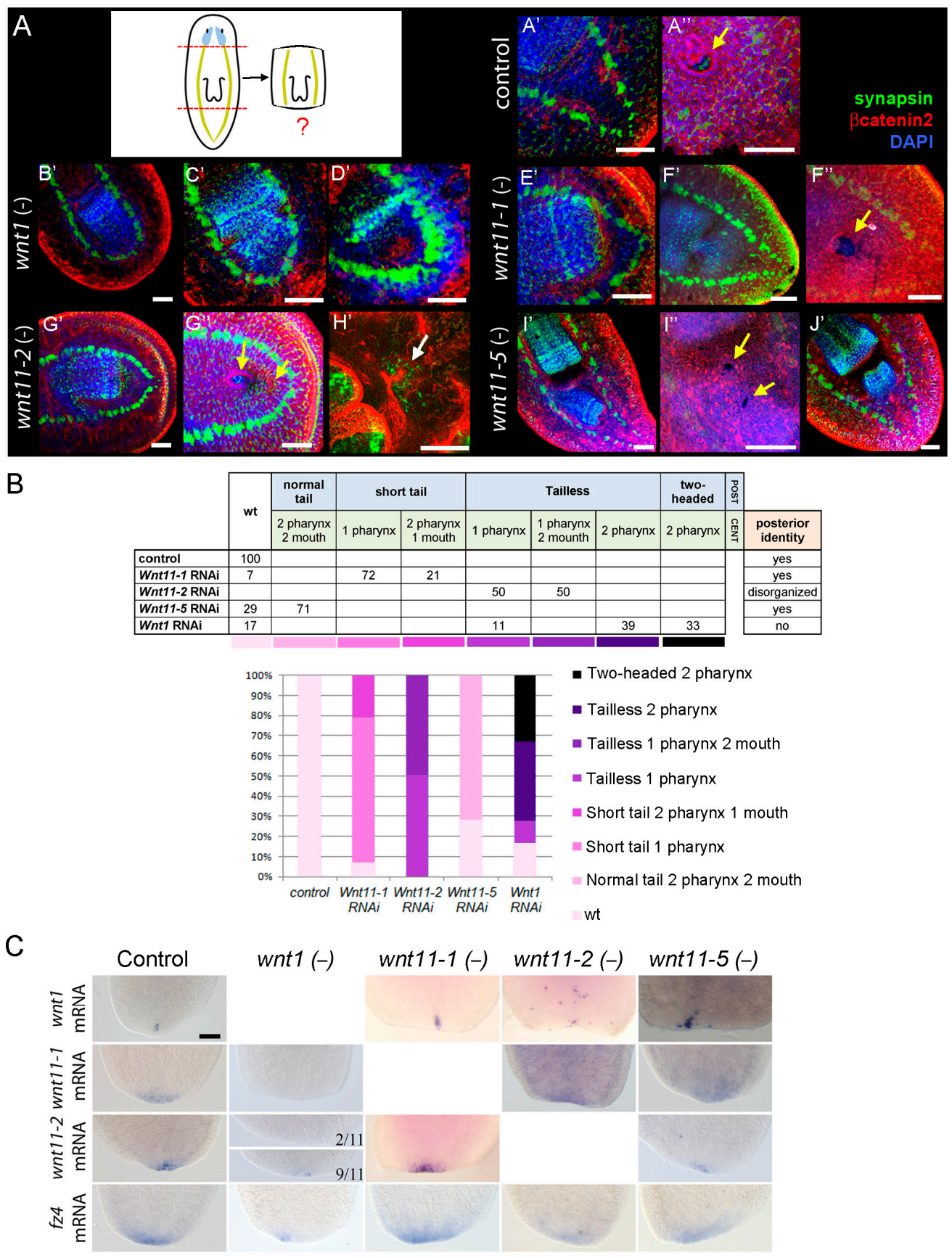

Figure 1. Each posterior wnt exerts a distinct function during planarian posterior regeneration. (A) Immunohistochemical analysis of planarian tail after silencing of Smed-wnt1 $\left(\mathbf{B}^{\prime}-\mathbf{D}^{\prime}\right)$,

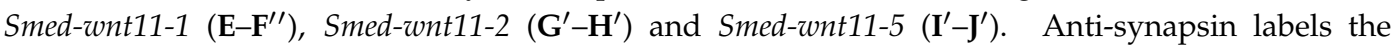
nervous system (green), anti- $\beta$ catenin1 labels adherent junctions (red), and nuclei are stained with DAPI (4',6-diamidino-2-phenylindole)(blue). $\mathbf{A}^{\prime \prime}, \mathbf{F}^{\prime \prime}, \mathbf{G}^{\prime \prime}$ and $\mathbf{I}^{\prime \prime}$ show a magnification of the plane corresponding to the mouth opening of $\mathbf{A}^{\prime}, \mathbf{F}^{\prime}, \mathbf{G}^{\prime}$ and $\mathbf{I}^{\prime}$, respectively (mouth openings are indicated with yellow arrows). A primordium of a second pharynx in Smed-wnt11-2 RNAi animals is shown in $\mathbf{H}^{\prime}$ (white arrow). Animals were fixed at 20 days of regeneration. All images correspond to confocal z-projections; (B) Quantification of the different phenotypes observed after silencing each posterior wnt. (The number of animals analyzed for each condition was at least $n=14$.). wt; wild type; (C) In situ hybridization analysis of the expression of posterior markers in 3-day regenerating posterior wnt RNAi. (The number of animals analyzed for each condition was at least $n=11$.). Anterior is left/up, posterior is right/down in (A); anterior is up, posterior is down in C. Scale bar: $100 \mu \mathrm{m}(\mathbf{A}, \mathbf{C})$. 


\subsection{Smed-wnt11-2 and Smed-wnt11-5 but not Smed-wnt11-1 Cooperate with Smed-wnt1 in Specifying Posterior Identity}

To study whether posterior wnts play a cooperative role in posterior specification and patterning, we silenced Smed-wnt1 (the only wnt that leads to shift of posterior to anterior identity upon silencing) simultaneously with Smed-wnt11-1, Smed-wnt11-2 or Smed-wnt11-5. The resulting phenotypes were analyzed by immunohistochemistry with anti-synapsin and anti- $\beta$ catenin 2 antibodies to visualize the nervous and digestive systems (Figure 2A). The phenotypes obtained after double inhibition were quantified and compared with those obtained after single inhibition of each posterior wnt (Figure 2A), allowing visualization of the degree of cooperation between the different posterior wnts in central and posterior specification. In these experiments, the penetrance of the phenotypes of single wnt RNAi was milder than in the experiments shown above, since half the amount of dsRNA was injected for each gene in order to maintain the total amount of dsRNA injected per animal (see Section 4.2). Quantification of the different phenotypes shows that simultaneous silencing of Smed-wnt1 together with Smed-wnt11-2 or Smed-wnt11-5 increased the number of "two-headed" planarians from 20\% in Smed-wnt1 RNAi planarians to $70 \%$ in the doubles [14]. In contrast, simultaneous silencing of Smed-wnt1 together with Smed-wnt11-1 decreased the frequency of "two-headed" planarians from $20 \%$ to $8 \%$. Interestingly, two new phenotypes not observed in the single inhibition experiment appeared in these experiments. Firstly, we observed "tailless" planarians with two pharynges in parallel, which is the addition of the suppression of the posterior identity after Smed-wnt1 silencing together with the appearance of an ectopic pharynx after Smed-wnt11-1. In addition, "tailless" planarians were observed with two pharynges in tandem and in the same orientation, which is the addition of the suppression of the posterior identity after Smed-wnt1 silencing and the duplication of the central identity produced by Smed-wnt11-5 silencing (Figure $2 \mathrm{~A}\left(\mathrm{~A}^{\prime}, \mathrm{B}^{\prime}\right)$ ). According to the phenotypes observed, analysis of the posterior marker $f z 4$ in the double RNAi planarians revealed a loss or reduction in Smed-wnt1/Smed-wnt11-2 and Smed-wnt1/Smed-wnt11-5 RNAi planarians (Figure 2B). Smed-wnt1/Smed-wnt11-1 RNAi animals also displayed a mild reduction of $f z 4$ expression, possibly due to the inhibition of Smed-wnt1. Taken together, these results demonstrate that Smed-wnt11-2 and Smed-wnt11-5, but not Smed-wnt11-1, cooperate with Smed-wnt1 in specifying posterior identity. The contribution of Smed-wnt11-2 in posterior specification could be predicted according to its requirement in single RNAi experiments. However, the contribution of Smed-wnt11-5 in posterior specification should be in cooperation with Smed-wnt1, since its inhibition alone never induces posterior defects. The possible cooperation between Smed-wnt11-1, Smed-wnt11-2 and Smed-wnt11-5 in the specification and patterning of the central region requires further attention.

A

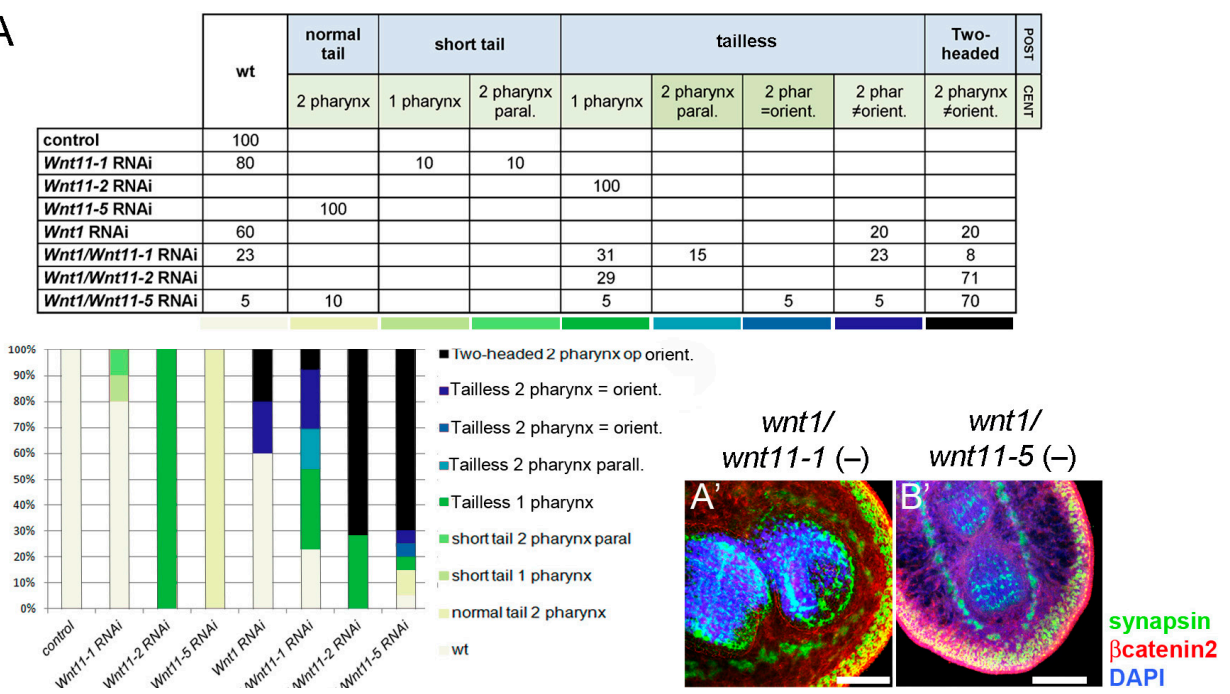

Figure 2. Cont. 


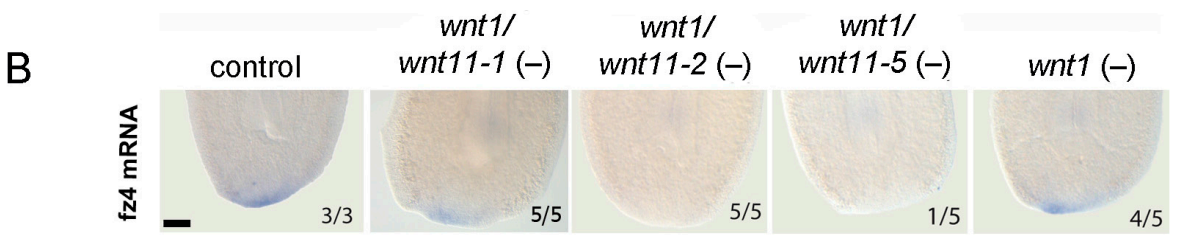

Figure 2. Cooperation of posterior wnts to specify posterior identity during regeneration. (A) Quantification of the different phenotypes observed after silencing each posterior wnt alone and Smed-wnt1 in combination with the other posterior wnts. Two new phenotypes appeared

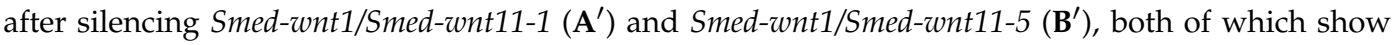
a "tailless" morphology next to the differentiation of a second pharynx alongside the original one. Animals were fixed at 20 days of regeneration. (The number of animals analyzed for single RNAi was $n=4-10$ and for double $n=7-20$.). $\mathbf{A}^{\prime}, \mathbf{B}^{\prime}$ images correspond to confocal z-projections; and (B) In situ hybridization analysis of the expression of posterior markers in 3-day regenerating animals in which Smed-wnt 1 was silenced together with the other posterior wnts. (The number of animals analyzed for each condition was $n=3-5$.). Anterior is left/up, posterior is right/down in $\mathbf{A}^{\prime}, \mathbf{B}^{\prime}$; anterior is up, posterior is down in (B). Scale bar: $100 \mu \mathrm{m}(\mathbf{A}, \mathbf{B})$.

\subsection{Silencing of All Posterior Wnts Together Is Insufficient to Transform Posterior Identity into Anterior during Homeostasis}

Bcatenin-dependent Wnt signaling is also required for the maintenance of posterior identity and pattern during planarian homeostasis, since Smed-Bcatenin 1 inhibition in intact planarians produces the appearance of ectopic eyes and brain in the posterior tip $[12,17,18]$. To analyze the possible cooperation between posterior wnts in the maintenance and pattern of the AP axis during homeostasis, we silenced them simultaneously and analyzed the resulting phenotypes after 6 weeks of inhibition. As a previous step, we silenced each wnt alone and showed that posterior eyes were not induced in any case (Figure S3). However, Smed-wnt11-1 and Smed-wnt11-2 RNAi planarians did show "tailless" phenotypes. RNAi of the four posterior wnts simultaneously produced an evident reduction of the tail, generating a strong "tailless" phenotype. In those animals, the pattern of the central region was also affected, and 3 types of central phenotypes could be distinguished: animals with two pharynges in opposite orientation, animals with a disorganized pharynx, and animals without a pharynx, due to its expulsion (Figure 3A,B). Despite the strong phenotype observed in posterior wnt RNAi planarians, the differentiation of ectopic anterior structures never occurred. The analysis of anterior and posterior identity markers corroborates the "tailless" phenotype, since RNAi planarians completely lost the expression of the posterior marker $f z 4$, and the anterior markers $s F R P$ [18] and notum [16] never appear in the posterior region (Figure 3C). Moreover, sFRP staining also revealed disorganization of the pharynx (Figure 3C).

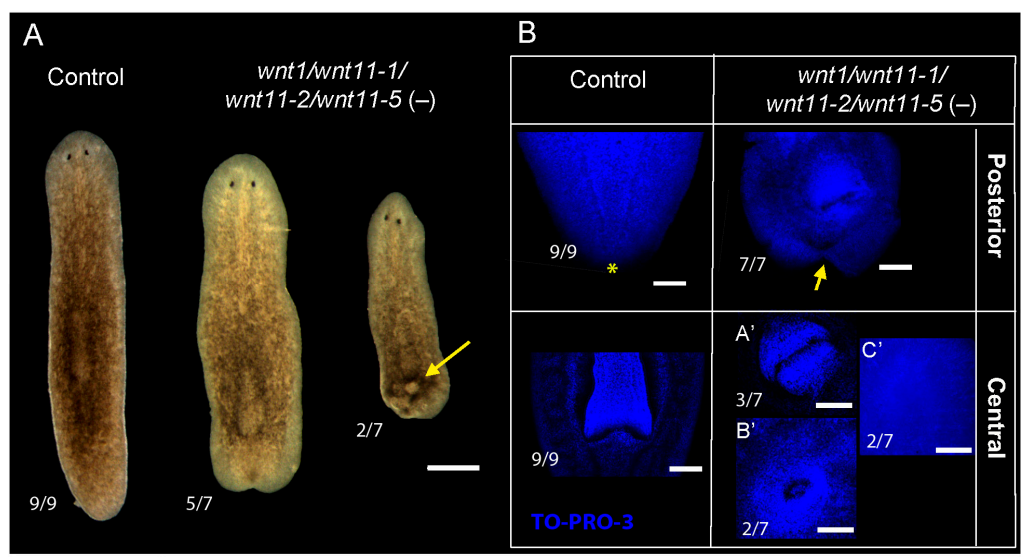

Figure 3. Cont. 


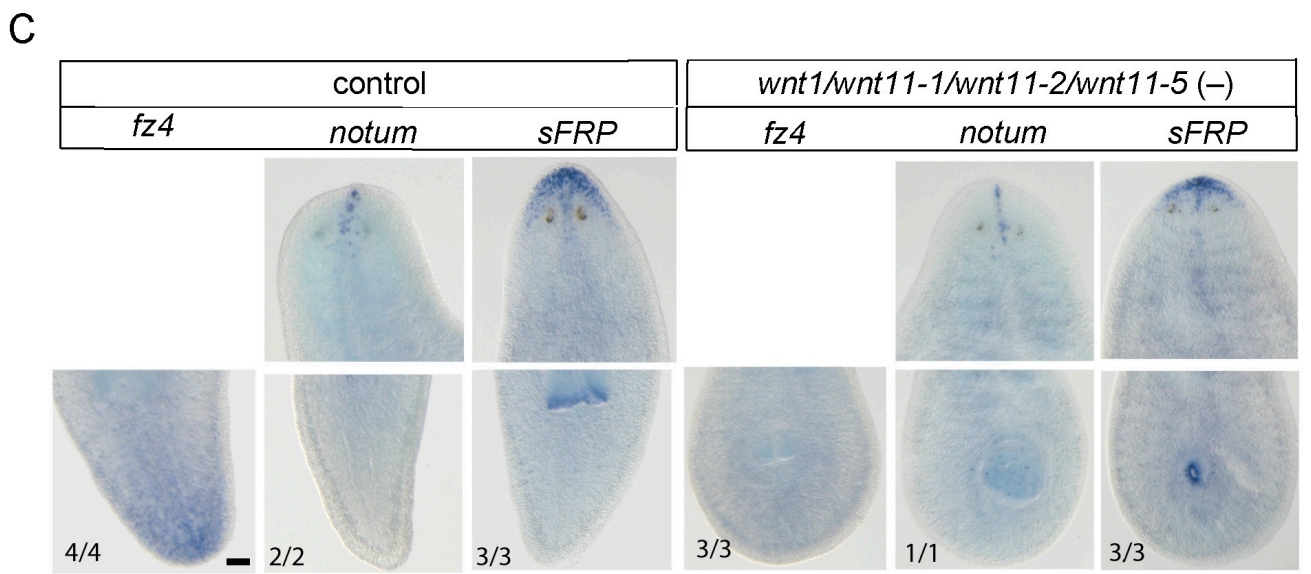

Figure 3. Silencing of all posterior wnts during homeostasis generates a strong "tailless" phenotype, without neither posterior nor anterior identity (A) After 6 rounds of Smed-wnt1/Smed-wnt11-1/ Smed-wnt11-2/Smed-wnt11-5 inhibition, planarians show a "tailless" phenotype in which the central region is also affected, since the pharynx cannot be maintained (yellow arrow points to a hole generated after the expulsion of the pharynx). (The number of animals analyzed for each condition was $n=7-9$ ); (B) TO-PRO-3 staining of the nucleus shows the "tailless" shape of RNAi planarians (yellow arrow) compared to controls (yellow asterisk), and the disorganization of the central region ( $\mathbf{A}^{\prime}$, two pharynges; $\mathbf{B}^{\prime}$, disorganized pharynx; $\mathbf{C}^{\prime}$, no pharynx, after expulsion). All images correspond to confocal z-projections; and (C) "Tailless" Smed-wnt1/wnt11-1/wnt11-2/wnt11-5 RNAi animals do not show expression of either posterior $(F z 4)$ or anterior ( $s F R P$, notum) markers in the posterior region. Anterior markers are normally expressed in the anterior region. Anterior is up, posterior is down in all images. Scale bar: $500 \mu \mathrm{m}(\mathbf{A}), 100 \mu \mathrm{m}\left(\mathbf{B}, \mathbf{A}^{\prime}, \mathbf{B}^{\prime}\right.$ and $\left.\mathbf{C}^{\prime}\right)$ and $100 \mu \mathrm{m}(\mathbf{C})$.

Taken together, these results show that disruption of the central and posterior regions in intact planarians is much stronger when silencing all posterior wnts simultaneously than when they are silenced individually, providing evidence of cooperation in the patterning of these regions. However, in contrast to the results reported for Smed-Bcatenin1 silencing [12], a shift of posterior identity to anterior was not observed under homeostatic conditions.

\section{Discussion}

Depending on the dose and time of inhibition, Smed-Bcatenin1 RNAi induces a gradual anteriorization of planarians, from "tailless" to "radial-like hypercephalized" animals. Consequently, it has been proposed that the graded activation of Smed- $\beta$ catenin 1 from posterior to anterior is responsible for specifying the whole AP axis in planarians [12,20]. However, the wnts responsible for the nuclear localization of Smed- $\beta$ catenin1 in such a broad domain remained mainly elusive. Until now, only the involvement of Smed-wnt1 in Smed- $\beta$ catenin1 nuclearization had been suggested, since it is the only wnt for which inhibition induces the appearance of a posterior head during posterior regeneration $[14,15]$. However, the strong anteriorization observed after Smed- $\beta$ catenin 1 silencing has never been observed following inhibition of any wnt. In this study, we analyzed the function of the four wnts which are expressed in the posterior part of planarians (posterior wnts) and explored the possibility that they cooperate to pattern planarian AP axis (Figure 4). Our results confirm that Smed-wnt1 is the only wnt for which inhibition leads to a shift in posterior polarity during regeneration, when posterior identity must be re-specified. Moreover, we reproduce the "tailless" phenotypes after inhibition of Smed-wnt11-2 [15,19], which also must exert a role in posterior specification, according to the decreased and disorganized pattern of posterior markers. In contrast, our results demonstrate that Smed-wnt11-1 and Smed-wnt11-5 are not required for posterior specification, since the tip of the tail in those RNAi animals regenerates normally and posterior markers are normally expressed. Interestingly, our data point to a role for Smed-wnt11-5 in the 
specification or patterning central identity, since Smed-wnt11-5 RNAi animals regenerate a second pharynx and mouth posteriorly to the pre-existing one. The shorter tail of Smed-wnt11-1 RNAi planarians could indicate a role for this wnt in the extension of the tail. Moreover, our data suggests that Smed-wnt11-1 could exert a direct role in the formation of the mouth, since it is expressed in this organ, and Smed-wnt11-1 RNAi planarians never duplicate the mouth despite the presence of two pharynges.

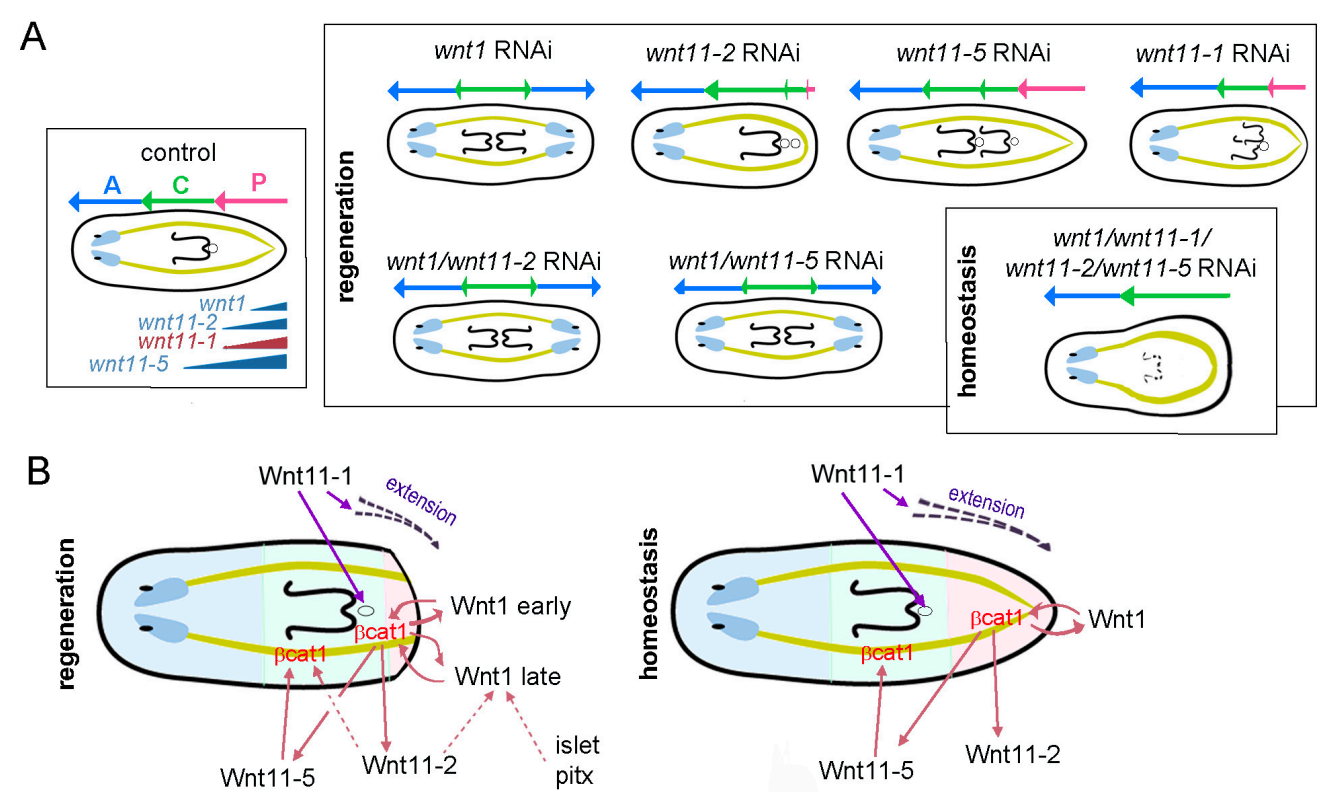

Figure 4. Summary and working model. (A) Scheme of the phenotypes generated after silencing the different posterior wnts (blue $\mathrm{A}$, anterior; green $\mathrm{C}$, central; pink $\mathrm{P}$, posterior). The strongest phenotype is represented; (B) Proposed model of the function of posterior wnts in central and posterior specification and patterning (in the planarian: blue is anterior; green is central and pink is posterior).

Based on the results obtained in this study, we hypothesize that Smed-wnt1, Smed-wnt11-2 and Smed-wnt11-5 could act in a $\beta$ catenin-dependent manner, nuclearizing Smed- $\beta$ catenin 1 in different domains along the AP axis (Figure 4B). Whereas Smed-wnt1 and Smed-wnt11-5 could be direct regulators of the $\beta$ catenin destruction complex in the posterior and central region, respectively, Smed-wnt11-2 could be modulating Smed- $\beta$ catenin1 activity indirectly, at least in the posterior region. This possibility is supported by the observation that Smed-wnt1 does not disappear but shows a disorganized pattern in Smed-wnt11-2 RNAi planarians (Figure 4B). At this point, it should be noted that two different stages of Smed-wnt1 expression occur during regeneration: an early Smed-wnt1 expression, which occurs during wounding and is stem-cell independent, and a late Smed-wnt1 expression, localized in the most posterior tip (the area which would correspond to the posterior organizer), that is stem-cell dependent [19]. We hypothesize that posterior identity is established by early Smed-wnt1 expression, which triggers the sustained activation of Smed- $\beta$ catenin1 in posterior regions through the subsequent activation of the late Smed-wnt1 expression (Figure 4B). Smed-wnt11-2, for which inhibition leads to "tailless" planarians, would be required for the proper expression pattern of the late Smed-wnt1. The concentration of Smed-wnt1 in the posterior tip would be essential for the establishment of the organizing region, which is responsible for growth and pattern rather than for identity specification. Additional factors, such as Smed-pitx or Smed-islet, could cooperate with Smed-wnt11-2, since their inhibition leads to suppression of late Smed-wnt1 expression and regeneration of "tailless" planarians [21,22]. It has been proposed that the "tailless" phenotype could also be the result of Smed-wnt11-2 acting in the establishment of the posterior midline [19]. In our view, the abolishment of the posterior midline goes together 
with the disruption of the posterior organizer. Smed-wnt11-1 RNAi animals regenerate a shorter tail showing a proper terminal identity. Moreover, their occasionally duplicated pharynx never locates in tandem, like in Smed-wnt11-5 or Smed-wnt1 RNAi planarians. For that reason, we hypothesize that Smed-wnt11-1 would not function in a Bcatenin-dependent manner but it would be involved in the non-canonical/ $\beta$ catenin-independent Wnt signaling, a well known mechanism to regulate migration and cell movement, which are the main morphogenetic processes required for tissue extension and epithelial rearrangements [6]. The possible non-canonical function of Smed-wnt11-1 and Smed-wnt11-2 compared to the Bcatenin-dependent function of Smed-wnt11-5 is further supported by their evolutionary origin, since phylogenetic analysis shows that Smed-wnt11-5 does not branch with Wnt11 but with the Wnt4 family [23]. Moreover, a wnt4 has been suggested to act in a ßcatenin-dependent manner in the platyhelminth Schistosoma [24]. Altogether, our results suggest that posterior wnts act in cooperation to provide a precise spatiotemporal control of the $\mathrm{AP}$ axis, from the pre-pharyngeal region to the tip of the tail.

The cooperation and integration of $\beta$ catenin-dependent and -independent Wnt signaling has been demonstrated to be essential also in the patterning of the AP neuroectoderm axis in sea urchin [25]. In cnidarians, it has been suggested that the patterning of the oral-aboral axis could be established by the cooperation between different Wnts, a "Wnt code", which would exert the function of the Hox code in bilatelians [26]. If the cooperation of posterior wnts is also required for maintenance of the AP pattern during homeostasis in planarians, then we expect that inhibition of the whole posterior wnt complement would lead to the abolishment of the identities from the pre-pharynx to the tail. Our results show that inhibition of posterior wnts during homeostasis one by one never induces the appearance of ectopic anterior structures but only generates mild "tailless" phenotypes. In contrast, inhibition of all posterior wnts together leads to a strong "tailless" phenotype, in which posterior markers disappear and also the central region is affected, since the

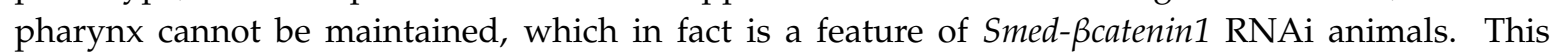
result confirms the hypothesis that posterior wnts cooperate to pattern the AP axis, including central and posterior regions. However, inhibition of the whole posterior wnt complement never induces the appearance of ectopic anterior structures, as occurs after Smed- $\beta$ catenin1 silencing. One reason could be that silencing all posterior wnts simultaneously affects not only the bcatenin-dependent but also the bcatenin-independent Wnt signaling, which could prevent cell tip specification. Further RNAi analysis with different combinations of posterior wnts should be performed. A second reason could be that RNAi inhibition of the secreted elements of the pathway is less efficient than inhibition of the intracellular element, particularly considering that we are silencing four genes simultaneously. However, it must be noted that silencing of Smed-wnt1 alone produces a strong anteriorization of planarians during regeneration but has no apparent phenotype during homeostasis. This observation could indicate that the signals which trigger posterior identity are different in the context of regeneration, when the posterior organizer must be re-specified, compared with the context of homeostasis, when the posterior organizer must be only maintained. A robust signaling network could underlie the maintenance of the posterior organizer (high levels of Smed- $\beta$ catenin1). Only the inhibition of Smed-Bcatenin1 itself or downstream elements, like Smed-teashirt [27], or the removal of the organizer after a posterior amputation, enables its re-specification towards a different fate.

\section{Experimental Section}

\subsection{Planarian Culture}

Planarians used in the presented experiments correspond to the clonal strain of S. mediterranea known as BCN-10 biotype. They were maintained as previously described [28]. Planarians used in these experiments were $4-6 \mathrm{~mm}$ length and were starved for 1 week before used for experiments. 


\subsection{RNAi Analysis}

Double-stranded RNAs (dsRNAs) used in these experiments were synthesized by in vitro transcription (Roche) as previously described [29]. dsRNA microinjections were performed in the digestive system of planarians following the standard protocol of a $3 \times 32 \mathrm{~nL} /$ injection of double-stranded (ds) RNA for three consecutive days before being amputated [29]. In regeneration experiments, 2 consecutive rounds of dsRNA injections were performed ( 1 round corresponds to 1 week, in which animals are injected on the first 3 days and amputated on the fourth). Animals were amputated transversally in 3 parts (heads, trunks and tails). In homeostasis experiments, 1 round of injection corresponds to 1 week in which dsRNA is injected on the first 3 days. Control animals were injected with dsRNA for the green fluorescent protein (GFP) sequence. In simultaneous gene-silencing experiments, the total amount of dsRNA injected for each gene and also the total amount of dsRNA injected in each animal was maintained constant by injecting the amount of GFP required.

\subsection{Whole-Mount in Situ Hybridization}

The RNA probes used in the present experiments were synthesized in vitro using Sp6 or T7 polymerase (Roche, Sant Cugat del Vallès, CAT, Spain) and DIG-modified ribonucleotides (Roche). Afterwards they were purified by ethanol precipitation and $7.5 \mathrm{M}$ ammonium acetate addition. For in situ hybridization, animals were killed with $\mathrm{HCl} 2 \%$, and fixed in Carnoy. An in situ Pro hybridization robot (Abimed/Intavis, Tübinguen, BW, Germany) was used for the in situ protocol, as previously described [30,31]. The temperature used for hybridizations was $56{ }^{\circ} \mathrm{C}$, and were carried out for $16 \mathrm{~h}$. A Leica MZ16F microscope (Leica Microsystems, Mannhiem, BW, Germany) was used to observe the samples. Images were captured with a ProgRes C3 camera from Jenoptik (Jena, TH, Germany).

\subsection{Immunostaining}

Immunostaining was carried out as described in previous studies [32]. The antibodies used in these experiments were: anti-synapsin (anti-SYNORF1,1:50, Developmental Studies Hybridoma Bank, Iowa City, IA, USA), anti-Smed- $\beta$-catenin2 (1:1000) [33] and anti- $\alpha$-tubulin (AA4, 1:20, Developmental Studies Hybridoma Bank). Alexa 488-conjugated goat anti-mouse (1:400, Molecular Probes, Waltham, MA, USA) and Alexa 568-conjugated goat anti-rabbit (1:1000, Molecular Probes) were used as a secondary antibodies. Nuclei were stained with DAPI (1:5000) or TO-PRO ${ }^{\circledR}-3$ (1:3000, Thermo Fisher Scientific, Waltham, MA, USA). A Leica TCS-SP2 (Leica Lasertechnik, Heidelberg, BW, Germany) adapted for an inverted microscope (Leitz DMIRB, Leica Lasertechnik, Heidelberg, BW, Germany) and a Leica TCS SPE (Leica Microsystems, Mannhiem, BW, Germany) were used to obtain confocal images. Representative confocal stacks for each experimental condition are shown.

Supplementary Materials: Supplementary materials can be found at http://www.mdpi.com/1422-0067/16/ 11/25970/s1.

Acknowledgments: We thank to E. Saló for suggestions and discussion of the manuscript. Monoclonal anti- SYNORF1 antibodies were obtained from the Developmental Studies Hybridoma Bank, developed under the auspices of the National Institute of Child Health and Human Development and maintained by the Department of Biological Sciences, University of Iowa, Iowa City, IA, USA. This work was supported by grant BFU2008-01544 from the Ministerio de Educación y Ciencia (MEC), Spain, and grant 2009SGR1018 (AGAUR). Miquel Sureda-Gómez received an FI fellowship from the Generalitat de Catalunya.

Author Contributions: Teresa Adell and Miquel Sureda-Gómez conceived and designed the experiments; Miquel Sureda-Gómez and Eudald Pascual-Carreras performed the experiments; Teresa Adell, Miquel Sureda-Gómez and Eudald Pascual-Carreras analyzed the data; Teresa Adell and Miquel Sureda-Gómez wrote the paper.

Conflicts of Interest: The authors declare no conflict of interest. 


\section{References}

1. Komiya, Y.; Habas, R. Wnt signal transduction pathways. Organogenesis 2008, 4, 68-75. [CrossRef] [PubMed]

2. Van Amerongen, R.; Nusse, R. Towards an integrated view of Wnt signaling in development. Development 2009, 136, 3205-3214. [CrossRef] [PubMed]

3. Mikels, A.J.; Nusse, R. Wnts as ligands: Processing, secretion and reception. Oncogene 2006, 25, 7461-7468. [CrossRef] [PubMed]

4. Petersen, C.P.; Reddien, P.W. Wnt signaling and the polarity of the primary body axis. Cell 2009, 139, 1056-1068. [CrossRef] [PubMed]

5. Mlodzik, M. Planar cell polarization: Do the same mechanisms regulate Drosophila tissue polarity and vertebrate gastrulation? Trends Genet. 2002, 18, 564-571. [CrossRef]

6. Wallingford, J.B.; Fraser, S.E.; Harland, R.M. Convergent extension: The molecular control of polarized cell movement during embryonic development. Dev. Cell 2002, 2, 695-706. [CrossRef]

7. Saló, E. The power of regeneration and the stem-cell kingdom: Freshwater planarians (Platyhelminthes). Bioessays 2006, 28, 546-559. [CrossRef] [PubMed]

8. Wagner, D.E.; Wang, I.E.; Reddien, P.W. Clonogenic neoblasts are pluripotent adult stem cells that underlie planarian regeneration. Science 2011, 332, 811-816. [CrossRef] [PubMed]

9. Baguna, J.; Saló, E.; Auladell, C. Regeneration and pattern formation in planarians. III. That neoblasts are totipotent stem cells and the cells. Development 1989, 107, 77-86.

10. Almuedo-Castillo, M.; Saló, E.; Adell, T. Dishevelled is essential for neural connectivity and planar cell polarity in planarians. Proc. Natl. Acad. Sci. USA 2011, 108, 2813-2818. [CrossRef] [PubMed]

11. Iglesias, M.; Almuedo-Castillo, M.; Aboobaker, A.A.; Saló, E. Early planarian brain regeneration is independent of blastema polarity mediated by the Wnt/ $\beta$-catenin pathway. Dev. Biol. 2011, 358, 68-78. [CrossRef] [PubMed]

12. Iglesias, M.; Gomez-Skarmeta, J.L.; Saló, E.; Adell, T. Silencing of Smed-ßcatenin1 generates radial-like hypercephalized planarians. Development 2008, 135, 1215-1221. [CrossRef] [PubMed]

13. Almuedo-Castillo, M.; Sureda-Gómez, M.; Adell, T. Wnt signaling in planarians: New answers to old questions. Int. J. Dev. Biol. 2012, 56, 53-65. [CrossRef] [PubMed]

14. Petersen, C.P.; Reddien, P.W. A wound-induced Wnt expression program controls planarian regeneration polarity. Proc. Natl. Acad. Sci. USA 2009, 106, 17061-17066. [CrossRef] [PubMed]

15. Adell, T.; Salò, E.; Boutros, M.; Bartscherer, K. Smed-Evi/Wntless is required for $\beta$-catenin-dependent and -independent processes during planarian regeneration. Development 2009, 136, 905-910. [CrossRef] [PubMed]

16. Petersen, C.P.; Reddien, P.W. Polarized notum activation at wounds inhibits Wnt function to promote planarian head regeneration. Science 2011, 332, 852-855. [CrossRef] [PubMed]

17. Petersen, C.P.; Reddien, P.W. Smed- $\beta$ catenin-1 is required for anteroposterior blastema polarity in planarian regeneration. Science 2008, 319, 327-330. [CrossRef] [PubMed]

18. Gurley, K.A.; Rink, J.C.; Sánchez Alvarado, A. $\beta$-Catenin defines head versus tail identity during planarian regeneration and homeostasis. Science 2008, 319, 323-327. [CrossRef] [PubMed]

19. Gurley, K.A.; Elliott, S.A.; Simakov, O.; Schmidt, H.A.; Holstein, T.W.; Alvarado, A.S. Expression of secreted Wnt pathway components reveals unexpected complexity of the planarian amputation response. Dev. Biol. 2010, 347, 24-39. [CrossRef] [PubMed]

20. Salo, E.; Adell, T.; Cebria, F. Gradients in planarian regeneration and homeostasis. Cold Spring Harb. Perspect. Biol. 2010, 2, 1-13.

21. März, M.; Seebeck, F.; Bartscherer, K. A Pitx transcription factor controls the establishment and maintenance of the serotonergic lineage in planarians. Development 2013, 140, 4499-4509. [CrossRef] [PubMed]

22. Hayashi, T.; Motoishi, M.; Yazawa, S.; Itomi, K.; Tanegashima, C.; Nishimura, O.; Agata, K.; Tarui, H. A LIM-homeobox gene is required for differentiation of Wnt-expressing cells at the posterior end of the planarian body. Development 2011, 138, 3679-3688. [CrossRef] [PubMed]

23. Riddiford, N.; Olson, P.D. Wnt gene loss in flatworms. Dev. Genes Evol. 2011, 221, 187-197. [CrossRef] [PubMed] 
24. Li, H.F.; Wang, X.B.; Jin, Y.P.; Xia, Y.X.; Feng, X.G.; Yang, J.M.; Qi, X.Y.; Yuan, C.X.; Lin, J.J. Wnt4, the first member of the Wnt family identified in Schistosoma japonicum, regulates worm development by the canonical pathway. Parasitol. Res. 2010, 107, 795-805. [CrossRef] [PubMed]

25. Range, R.C.; Angerer, R.C.; Angerer, L.M. Integration of canonical and noncanonical wnt signaling pathways patterns the neuroectoderm along the anterior-posterior axis of sea urchin embryos. PLoS Biol. 2013, 11, 1001467. [CrossRef] [PubMed]

26. Guder, C.; Philipp, I.; Lengfeld, T.; Watanabe, H.; Hobmayer, B.; Holstein, T.W. The Wnt code: Cnidarians signal the way. Oncogene 2006, 25, 7450-7460. [CrossRef] [PubMed]

27. Reuter, H.; März, M.; Vogg, M.C.; Eccles, D.; Grífol-Boldú, L.; Wehner, D.; Owlarn, S.; Adell, T.; Weidinger, G.; Bartscherer, K. $\beta$-Catenin-dependent control of positional information along the ap body axis in planarians involves a teashirt family member. Cell Rep. 2015, 10, 253-265. [CrossRef] [PubMed]

28. Fernandéz-Taboada, E.; Moritz, S.; Zeuschner, D.; Stehling, M.; Schöler, H.R.; Saló, E.; Gentile, L. Smed-SmB, a member of the LSm protein superfamily, is essential for chromatoid body organization and planarian stem cell proliferation. Development 2010, 137, 1055-1065. [CrossRef] [PubMed]

29. Sánchez Alvarado, A.; Newmark, P. Double-stranded RNA specifically disrupts gene expression during planarian regeneration. Proc. Natl. Acad. Sci. USA 1999, 96, 5049-5054. [CrossRef] [PubMed]

30. Umesono, Y.; Watanabe, K.; Agata, K. A planarian orthopedia homolog is specifically expressed in the branch region of both the mature and regenerating brain. Dev. Growth Differ. 1997, 39, 723-727. [CrossRef] [PubMed]

31. Molina, M.D.; Saló, E.; Cebrià, F. The BMP pathway is essential for re-specification and maintenance of the dorsoventral axis in regenerating and intact planarians. Dev. Biol. 2007, 311, 79-94. [CrossRef] [PubMed]

32. Ross, K.G.; Omuro, K.C.; Taylor, M.R.; Munday, R.K.; Hubert, A.; King, R.S.; Zayas, R.M. Novel monoclonal antibodies to study tissue regeneration in planarians. BMC Dev. Biol. 2015, 15, 2. [CrossRef] [PubMed]

33. Chai, G.; Ma, C.; Bao, K.; Zheng, L.; Wang, X.; Sun, Z.; Salò, E.; Adell, T.; Wu, W. Complete functional segregation of planarian $\beta$-catenin-1 and -2 in mediating Wnt signaling and cell adhesion. J. Biol. Chem. 2010, 285, 24120-24130. [CrossRef] [PubMed]

(C) 2015 by the authors; licensee MDPI, Basel, Switzerland. This article is an open access article distributed under the terms and conditions of the Creative Commons by Attribution (CC-BY) license (http://creativecommons.org/licenses/by/4.0/). 